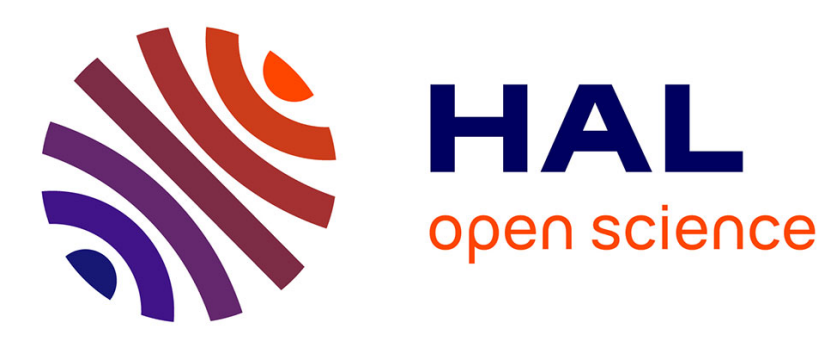

\title{
A novel combined PAPR reduction and channel estimation approach for OFDM systems
}

Anh Tai Ho, Jean-François Hélard, Youssef, Joseph Nasser, Yves Louët

\section{To cite this version:}

Anh Tai Ho, Jean-François Hélard, Youssef, Joseph Nasser, Yves Louët. A novel combined PAPR reduction and channel estimation approach for OFDM systems. IEEE International Symposium on Broadband Multimedia Systems and Broadcasting (ISBMSB'10), Mar 2010, Shanghai, China. hal00491661

\section{HAL Id: hal-00491661 \\ https://hal.science/hal-00491661}

Submitted on 14 Jun 2010

HAL is a multi-disciplinary open access archive for the deposit and dissemination of scientific research documents, whether they are published or not. The documents may come from teaching and research institutions in France or abroad, or from public or private research centers.
L'archive ouverte pluridisciplinaire HAL, est destinée au dépôt et à la diffusion de documents scientifiques de niveau recherche, publiés ou non, émanant des établissements d'enseignement et de recherche français ou étrangers, des laboratoires publics ou privés. 


\title{
A novel combined PAPR reduction and channel estimation approach for OFDM systems
}

\author{
Anh Tai HO, Jean-François HELARD, Youssef NASSER and Yves LOUËT \\ Institute of Electronics and Telecommunications of Rennes, UMR CNRS 6164 \\ INSA de Rennes, 20 avenue des Buttes de Coësmes, 35043 Rennes, France \\ Email : \{anh-tai.ho, jean-francois.helard, youssef.nasser\} @insa-rennes.fr, yves.louet@ rennes.supelec.fr
}

\begin{abstract}
In this paper, a novel combination of peak-to-average power ratio (PAPR) reduction and channel estimation techniques for orthogonal frequency division multiplexing (OFDM) systems is addressed. In order to reduce the spectrum efficiency loss due the insertion of dedicated pilots for PAPR reduction issues, we propose to use some pilots dedicated for channel estimation to reduce the PAPR value. These pilots follow particular laws which allow their blind detection at the receiving side and avoid sending side information. At the receiver, based on these properties, the pilots are detected and channel estimation is then performed. The proposed laws operate in discrete domain to mitigate the performance degradation due to residual estimation error in continuous domain. Simulation results performed using the new DVB-T2 standard parameters show that the proposed approach gives better performance and higher spectral efficiency when compared to conventional Tone Reservation (TR) method.
\end{abstract}

Index Terms- DVB-T2, OFDM, PAPR, tone reservation, channel estimation.

\section{INTRODUCTION}

Orthogonal Frequency Division Multiplexing (OFDM) has been the subject of numerous dissertations in recent years, mainly due to its several advantages for mobile wireless communications such as high data rate transmission with high bandwidth efficiency, exceptional robustness to multi-path fading and low implementation complexity. It has been adopted in several standards such as IEEE 802.11a, IEEE 802.16a, ETSI HIPERLAN/2 and DVB [1-2].

However, OFDM system is not without its limitations [2]. The composite time signal in OFDM system is formed from the linear addition of independently modulated subcarriers. As a result, when the number of subcarriers is large, high amplitude peaks appear in the OFDM signal which induces a large Peakto-Average Power Ratio (PAPR). Usually, the OFDM signal passes through a non-linear power amplifier before its transmission over the channel. High PAPR value implies sophisticated high power amplifier (HPA) operating on a very large linear range at the transmitter. Otherwise, the nonlinearity of the HPA leads to in-band distortion, which increases the bit error rate (BER) of the system, and the out-of-band (OOB) distortion, which introduces high adjacent channel interference.

Various approaches have been proposed as resumed in [3] to mitigate the PAPR of an OFDM signal. Among them, clipping and filtering technique is easy to implement. However, this scheme decreases the system performance since clipping is a nonlinear process [4]. An alternative method is based on coding, in which a data sequence is embedded in a longer sequence and only a subset of all these possible sequences is used in order to exclude patterns generating high PAPR [5]. Moreover, other methods such as partial transmit sequence [6], selected mapping [7] and interleaving [8] are also proposed. The main drawback of these methods is the necessity to transmit side information (SI) to the receiver, resulting in some loss of throughput efficiency. Some methods recently proposed do not need this SI transmission [9-10]. Indeed, the active constellation extension (ACE) method is proposed in [9]. It reduces PAPR by changing the constellation of the signal without modifying the minimum distance. The tone reservation (TR) method is proposed by Tellado [10] which uses allocated subcarriers to generate additional signal that minimizes PAPR. These two methods have been already adopted for DVB-T2, the evolution of DVB-T standard. However, ACE technique increases the transmit signal power and its performance is rather restricted for a modulation with a large constellation size, while TR technique reduces the spectrum efficiency by the dedication process.

The minimization of PAPR in the TR method can be formulated as a convex optimization problem, which is modelized as a second order cone programming (SOCP) [11]. In this paper, we continue the work of [11] by investigating the TR method. The contribution of this paper is multifold. First, instead of using dedicated pilots for PAPR reduction, we propose to use existing pilots dedicated for channel estimation. In this way, we avoid the use of TR pilots and improve spectral efficiency of the system. Second, in order to allow their recovery serving for channel estimation process at the receiver, these pilots have to follow particular laws. Additive law and multiplicative law are then proposed and investigated in this work. At the receiver, laws relating pilots at the transmitter are applied to detect and estimate transmitted pilots. Since the detection and the estimation of these pilots in continuous domain cause degradation, we propose to apply these laws in discrete domain. Third, we show by simulations the validity of our technique using DVB-T2 standard parameters. Its performance is compared to the SOCP technique proposed in [11] and the Walsh-Hadamard sequences technique proposed in [12].

This paper is organized as follows. Section II briefly presents the OFDM system model and the PAPR statistics. In section III, a novel combined PAPR reduction and channel 
estimation approach is presented. Simulation results and discussions are addressed in section IV. Finally, conclusions are drawn in section $\mathrm{V}$.

\section{OFDM SIGNAL AND PAPR}

Let $X=\left[X_{0} \ldots X_{N-1}\right]$ be a sequence of complex symbols and $x=\left[x_{0} \ldots x_{N-1}\right]$ be its inverse Fourier transform. The OFDM baseband signal is expressed as

$$
x(t)=\frac{1}{\sqrt{N}} \sum_{k=0}^{N-1} X_{k} e^{\frac{j 2 \pi k t}{T N}} \quad 0 \leq t<N T
$$

where $j=\sqrt{-1}, N$ denotes the number of subcarriers and $T$ is the original complex signal duration. In practice, only $N L$ equidistant samples of $x(t)$ are considered, where $L$ represents the over-sampling factor. The over-sampled signal is then given by

$$
x[n / L]=\frac{1}{\sqrt{N}} \sum_{k=0}^{N-1} X_{k} e^{\frac{j 2 \pi n k}{L N}} \quad \forall n \in[0, \ldots, N L-1]
$$

When the over-sampling factor $\mathrm{L}$ is large enough, the PAPR of the OFDM signal is defined as the ratio of its maximum power divided by its average power. It is expressed as [13]

$$
\operatorname{PAPR}\{x(t)\} \approx \operatorname{PAPR}\left\{x_{L}, L \geq 4\right\}=\frac{\max _{n}\left|x_{n}\right|^{2}}{E\left\{\left|x_{L}\right|^{2}\right\}}
$$

where $x_{L}=Q_{L} X_{L}, \quad X_{L}$ is the zero-padded vector of $X$, $E\{$.$\} denotes expectation operation and Q_{L}$ is the inverse discrete Fourier transform matrix of size $N L . Q_{L}$ is given by

$$
Q_{L}=\frac{1}{\sqrt{N}}\left[\begin{array}{cccc}
1 & 1 & \ldots & 1 \\
1 & e^{\frac{j 2 \pi}{N L} 1.1} & \ldots & e^{\frac{j 2 \pi}{N L} 1 .(N L-1)} \\
\vdots & \vdots & \ldots & \vdots \\
1 & e^{\frac{j 2 \pi}{N L}(N L-1) .1} & \ldots & e^{\frac{j 2 \pi}{N L}(N L-1) .(N L-1)}
\end{array}\right]
$$

In this study, the PAPR performance is evaluated using the complementary cumulative distribution function (CCDF). It is defined by the probability that the PAPR value exceeds a given threshold $\gamma$. For $L=1$, it can be expressed as [11]

$$
\begin{aligned}
C C D F_{P A P R}\{x(t)\} & =\operatorname{Pr}[\operatorname{PAPR}\{x(t)\}>\gamma, L=1] \\
& \approx 1-\left(1-e^{-\gamma}\right)^{N}
\end{aligned}
$$

It is demonstrated in [14] that real PAPR can be approximated when $L=4$ by

$$
\begin{aligned}
C C D F_{P A P R}\{x(t)\} & =\operatorname{Pr}[\operatorname{PAPR}\{x(t)\}>\gamma, L=4] \\
& \approx 1-\left(1-e^{-\gamma}\right)^{2.8 N}
\end{aligned}
$$

For simulations, we consider $L=4$ and used this approximation as a reference PAPR value of the original OFDM signal.

\section{NOVEL COMBINED PAPR REDUCTION WITH CHANNEL ESTIMATION APPROACH}

\section{A. PAPR reduction}

In this section, we propose to use the pilots dedicated to channel estimation for PAPR reduction. Let's consider $P=\left[p_{0} \ldots p_{M-1}\right]$ be the set of $\mathrm{M}$ pilot positions dedicated to channel estimation to be used for PAPR reduction, and $C=\left[C_{0} \ldots C_{M-1}\right]$ be the set of $\mathrm{M}$ pilots transmitted on these positions. Then, $\mathrm{N}$ modulated symbols $\left\{S_{k}\right\}_{k=0 \ldots N-1}$ in the frequency domain are expressed as

$$
S_{k}=\left\{\begin{array}{cc}
C_{k} & \text { if } k \in P \\
X_{k} & \text { if } n o t
\end{array}\right.
$$

The OFDM baseband signal becomes

$$
s_{L}=Q_{L} S_{L}=Q_{L}\left(C+X_{k}\right)=x_{L}+Z_{L} C
$$

where $Z_{L}$ is given by

$$
Z_{L}=\frac{1}{\sqrt{N}}\left[\begin{array}{cccc}
1 & 1 & \ldots & 1 \\
1 & e^{\frac{j 2 \pi}{N L} p_{1} \cdot 1} & \ldots & e^{\frac{j 2 \pi}{N L} p_{1} \cdot(N L-1)} \\
\vdots & \vdots & \ldots & \vdots \\
1 & e^{\frac{j 2 \pi}{N L} p_{M} \cdot 1} & \ldots & e^{\frac{j 2 \pi}{N L} p_{M} \cdot(N L-1)}
\end{array}\right]
$$

At the transmitter, reducing PAPR turn out to the research of $C$ minimizing $\operatorname{PAPR}\left\{s_{L}\right\}$. In continuous domain, i.e. $C \subset C^{M}$, this is formulated as a SOCP problem [11]. At the receiver, $C$ has to be recovered in order to allow channel estimation operation. So, these pilots must follow particulars law. We consider two cases

- Additive law:

$$
\begin{aligned}
& C_{k+1}=C_{k}+\Omega \quad \forall k \in[0, \ldots, M-2] \\
& \text { then } C_{k}=C_{0}+k \Omega
\end{aligned}
$$

- Multiplicative law:

$$
\begin{aligned}
& C_{k+1}=C_{k} \times \Omega \quad \forall k \in[0, \ldots, M-2] \\
& \text { then } C_{k}=\Omega^{k} C_{0}
\end{aligned}
$$

where $\Omega$ denotes step between two consecutive pilots. 
In comparison with [11], this operation corresponds to an additional constraint on SOCP problem. At the receiver, estimating $C$ and $\Omega$ in continuous domain can cause degradation of the system performance due to residual estimation error. We propose to perform this in discrete domain. The laws become:

- Additive law:

$$
\begin{aligned}
& C_{k}=C_{0}+k \Omega \quad \forall k \in[0, \ldots, M-2] \\
& \text { with } C_{0}=\lambda e^{j \phi} \text { and } \Omega=\eta e^{j \Delta} \\
& \text { then } C_{k}=\lambda e^{j \phi}+k \eta e^{j \Delta}
\end{aligned}
$$

- Multiplicative law:

$$
\begin{aligned}
& C_{k}=\Omega^{k} C_{0} \quad \forall k \in[0, \ldots, M-2] \\
& \text { with } \quad C_{0}=\lambda e^{j \phi} \text { and } \Omega=e^{j \Delta} \\
& \text { then } C_{k}=\lambda e^{j(\phi+k \Delta)}
\end{aligned}
$$

where $\lambda, \eta, \phi$ and $\Delta$ are in discrete domain. "Discrete domain" means that instead of searching SOCP solution in $C$ space, we search it in a predefined discrete set.

At the receiver, $C$ and $\Omega$ are detected and estimated. By quantifying them in discrete domain, $\lambda, \eta, \phi$ and $\Delta$ can be simply recovered.

\section{B. Pilot recovery and channel estimation}

Assuming a complex multiplicative channel, the received symbols in frequency domain are expressed as

$$
R^{l}=H^{l} \times S^{l}+W^{l}
$$

where $l$ refers to OFDM symbol index, $H^{l}$ denotes the $N \times N$ matrix containing the complex channel attenuation per subcarrier and $W^{l}$ is the additive white Gaussian noise vector.

Pilot recovery and channel estimation procedures are performed in two steps:

- Determine the transmitted pilots $\hat{C}^{l}$

- Estimate the actual channel $\hat{H}^{l}$

In order to accomplish the first step, we assume that the channel slightly change between two successive OFDM symbols, i.e. $\hat{H}^{l-1} \approx \hat{H}^{l}$. Then, we have

$$
\bar{C}_{k}^{l}=\frac{R_{k}^{l}}{\hat{H}_{k}^{l-1}} \quad \forall k \in P
$$

Since $C_{k}^{l}$ are in discrete domain, $\hat{C}_{k}^{l}$ can be estimated from $\bar{C}_{k}^{l}$ by a simple quantification operation.
For the second step, the channel response in the frequency domain is estimated at pilot positions as follows:

$$
\hat{H}_{k}^{l}=\frac{R_{k}^{l}}{\hat{C}_{k}^{l}} \quad \forall k \in P
$$

Then, channel frequency response for all subcarriers can be classically deduced by interpolation based on Wiener filtering.

\section{Simulations AND DisCUSSION}

Simulation results are performed using DVB-T2 parameters. Some of the main parameters are resumed in table 1. The PAPR parameters of the proposed method, $\lambda, \eta, \phi$ and, are selected according to the simulation results. They will be specified in each scenario.

TABLE I. SIMULATION PARAMETERS, EXTRACTED FROM DVB-T2 STANDARD.

\begin{tabular}{|l|l|}
\hline Mode & 2K \\
\hline Number of subcarriers & NFFT $=2048$ \\
\hline Guard Interval length & $\mathrm{GI}=1 / 8$ \\
\hline Modulation & $16-\mathrm{QAM}$ \\
\hline Coding rate & $\mathrm{CR}=1 / 2$ \\
\hline Over-sampling factor & $\mathrm{L}=4$ \\
\hline $\begin{array}{l}\text { Number of subcarriers used } \\
\text { for PAPR reduction }\end{array}$ & $\mathrm{M}=8,16,32$ \\
\hline
\end{tabular}

A. Comparison between additive and multiplicative laws

Firstly, we compare the performance of additive and multiplicative laws. Let's consider SOCP solution in the continuous domain with $M=8$. $\phi$ varies from 0 to $2 \pi$ with a step of $\pi / 8$, while $\Delta$ varies from 0 to $2 \pi$ with variable steps, of $\pi / 2$, $\pi / 4, \pi / 8$ and $\pi / 16$. The results are presented in figure 1 .

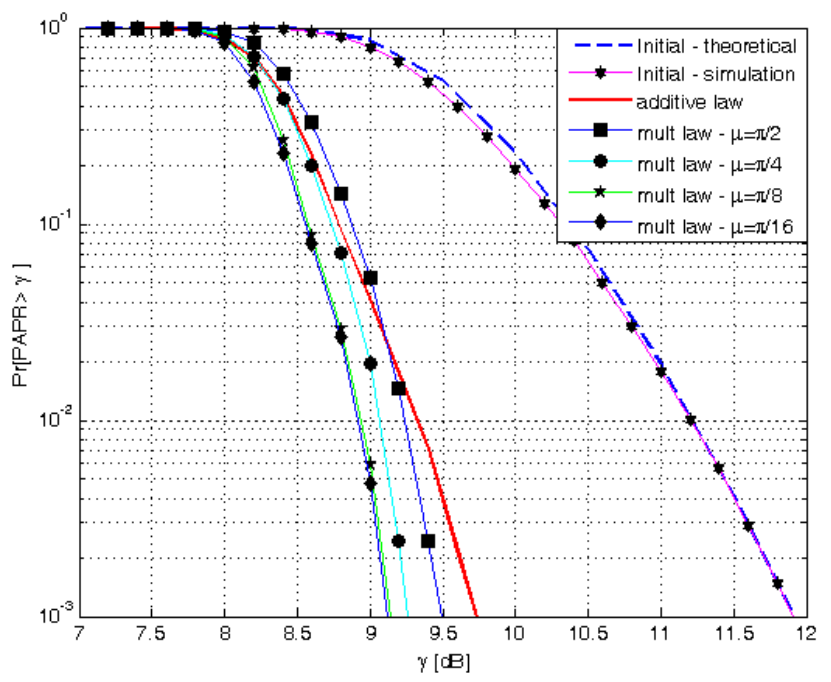

Figure 1. CCDF performance of additive and multiplicative laws, SOCP solution.

Multiplicative law gives better performance than the additive law. This is because in multiplicative law, all pilot 
subcarriers are modulated with the same power. Moreover, multiplicative law is performed with $\phi$ and $\Delta$ in the discrete domain while additive law is performed in continuous domain. So in the discrete domain, this difference is still more important. Then, the multiplicative law presents better performance than the additive law in discrete domain. For the rest of this paper, we investigate the evaluation of the performance for multiplicative law in discrete domain.

\section{B. Multiplicative law performance in discrete domain}

We consider now multiplicative law in the discrete domain. Firstly, we present CCDF performance for fixed value of $\lambda$ and for $\phi$ and $\Delta$ varying from 0 to $2 \pi$ with a step of $\pi / 8$. The number of subcarriers used for PAPR reduction is always $\mathrm{M}=8$. In this case, reducing PAPR is not a SOCP problem anymore but becomes a research of the optimum combination minimizing PAPR. At the receiver, pilots are not recovered by a systematic research (as the one presented in [12]) but by a detection and estimation algorithm. These results are shown in figure 2.

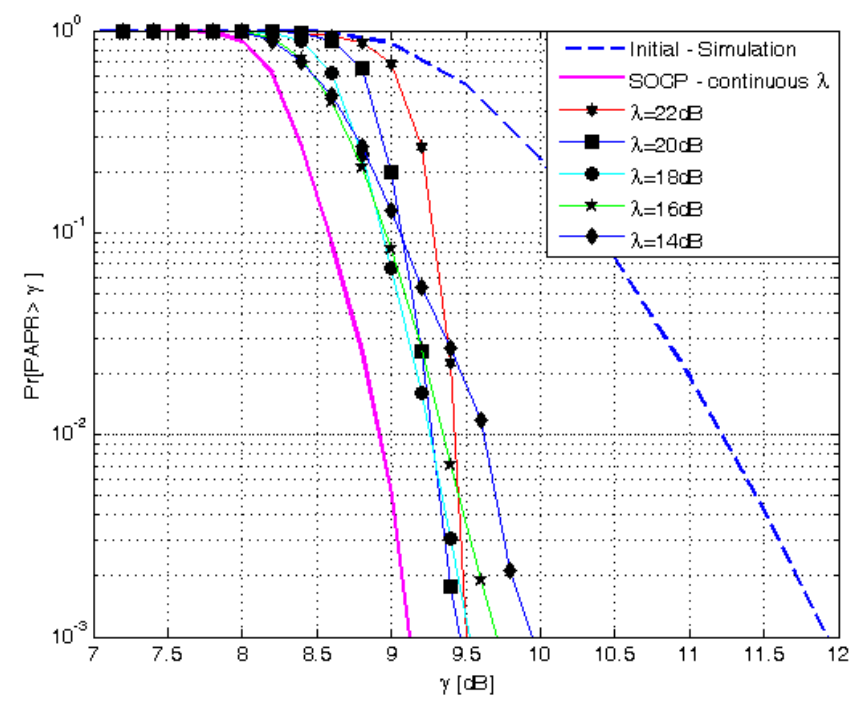

Figure 2. CCDF performance of multiplicative law in discrete domain.

We note that the performance in discrete domain is slightly worse than the one of SOCP solution and it depends on the value of $\lambda$. Then, we present in figure 3 the PAPR gain in terms of CCDF, in comparison with theoretical PAPR, as a function of $\lambda . \phi$ and $\Delta$ varying from 0 to $2 \pi$ with step of $\pi / 8, \pi / 16$ and $\pi / 32$, are considered. This gain is evaluated at $\mathrm{CCDF}=10^{-3}$. It is also compared with the SOCP solution for multiplicative law ( $\lambda$ continuous) and the Walsh-Hadamard sequences method presented in [12], named OPS method.

The system performance improves when the step of $\phi$ and $\Delta$ becomes smaller. This is because the number of pilot combinations increases when this step decreases. For each value of this step, there exists one optimum value of $\lambda$ for which the PAPR gain in terms of CCDF reaches the maximum. Besides, we note that the performance of the proposed method is much better than the one of the OPS method proposed in [12].

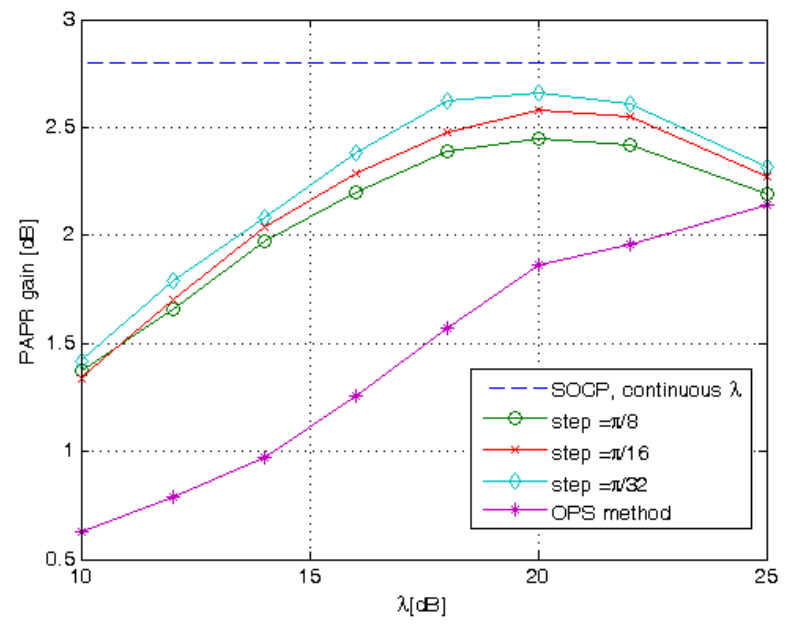

Figure 3. CCDF performance as a function of $\lambda, \mathrm{M}=8$.

Finally, the performance in terms of PAPR gain is presented in figure 5, for $M=8,16$ and 32, for $\phi$ and $\Delta$ varying from 0 to $2 \pi$ with step of $\pi / 8, \pi / 16$ and $\pi / 32$, as a function of $\lambda$.

TABLE II. PERFORMANCE SUMMARY OF DIFFERENT METHODS, DVB-T2 PARAMETERS, 2K MODE, 16-QAM MODULATION.

\begin{tabular}{|c|c|c|c|}
\hline \multicolumn{2}{|c|}{ Methods } & $\begin{array}{c}\Delta \mathrm{G} \\
(\mathrm{dB})\end{array}$ & $\begin{array}{c}\lambda_{\text {opt }} \\
(\mathrm{dB})\end{array}$ \\
\hline \multirow{4}{*}{$\begin{array}{c}\text { Proposed } \\
\text { method }\end{array}$} & $\mathrm{M}=8, \mu=\pi / 16$ & 2.58 & 20 \\
\cline { 2 - 4 } & $\mathrm{M}=8, \quad \mu=\pi / 32$ & 2.66 & 20 \\
\cline { 2 - 4 } & $\mathrm{M}=16, \mu=\pi / 16$ & 2.07 & 14 \\
\cline { 2 - 4 } & $\mathrm{M}=16, \mu=\pi / 32$ & 2.17 & 14 \\
\cline { 2 - 4 } & $\mathrm{M}=32, \mu=\pi / 16$ & 1.61 & 6 \\
\cline { 2 - 4 } & $\mathrm{M}=32, \mu=\pi / 32$ & 1.88 & 8 \\
\hline \multirow{3}{*}{$\begin{array}{c}\text { Gradient } \\
\text { method }\end{array}$} & $\mathrm{CLIP}=3 \mathrm{~dB}$ & 1.62 & - \\
\cline { 2 - 4 } & $\mathrm{CLIP}=5 \mathrm{~dB}$ & 2.01 & - \\
\hline
\end{tabular}

The PAPR gain performance slightly decreases when $M$ increases, but we note that the optimum value of $\lambda$ also decreases when $\mathbf{M}$ increases. This is important because it means that we can reduce pilot's power when increasing the number of subcarriers used for PAPR reduction.

Simulations results also show that the channel response is well estimated using this approach. As a consequence, this method allows avoiding the use of pilots dedicated for PAPR reduction, as TR pilots in DVB-T2 standard, improving also spectral efficiency.

\section{CONCLUSIONS}

In this paper, we have proposed a novel combined PAPR reduction and channel estimation method. Pilots dedicated for channel estimation have been used for both PAPR reduction and channel estimation purposes. These pilots followed 
particular laws in order to allow their detection at the receiver. Additive and multiplicative laws have been then investigated. By operating these laws in discrete domain, the system performance is improved because residual estimation error in continuous domain is avoided. Simulations showed that in discrete domain, multiplicative law is much better than additive law. Performance of the proposed method with multiplicative law in discrete domain was than evaluated using DVB-T2 parameters. Simulations shoed that it can achieve until $2.7 \mathrm{~dB}$ in terms of PAPR gain. In comparison with DVB-T2 standard, proposed method presented better PAPR gain than the gradient method initially proposed in DVB-T2. In terms of spectral efficiency, proposed method avoided the use of dedicated pilots, which are equal to $1 \%$ of subcarriers in DVB-T2 standard specifications, improving also spectral efficiency of the system.

\section{REFERENCES}

[1] U. Reimers, "Digital Video Broadcasting", IEEE Com.. Mag., vol. 36, no. 10, pp. 104-110, June 1998.

[2] R. Van Nee and R. Prasad, "OFDM for Wireless Multimedia Communications", Artech House Publisher, March 2000.

[3] S. H. Han, and J. H. Lee, "An overview of Peak-to-Average Power Ratio reduction techniques of multicarrier transmission", IEEE Wireless Com., vol. 12, no. 2, pp. 56-65, April 2005.

[4] X. Li and and J. L. Cimini, "Effect of Clipping and Filtering on the performance of OFDM", IEEE Com. Letters, vol. 2, no. 5, pp. 131-133, May 1998.

[5] A. E. Jones, T. A. Wilkinson and S. K. Barton, "Block coding scheme for reduction of Peak to Mean envelope Power Ratio of Multicarrier
Transmission scheme", IEEE Com. Letters, vol. 32, no. 22, pp. 20982099, Dec. 1994.

[6] S. H. Muller and J. B. Huber, "OFDM with reduced Peak-to-Average Power Ratio by Optimum Combination of Partial Transmit Sequences", Elec. Letters, vol. 32, no. 5, pp. 368-369, Feb. 1997.

[7] R. W. Bauml, R. F. H. Fisher and J. B. Huber, "Reducing the Peak-toAverage Power Ratio of multicarrier Modulation by Selected Mapping", Elec. Letters, vol. 32, no. 22, pp. 2056-2057, Oct 1996.

[8] A. D. S. Jayalath and C. Tellambura, "Reducing the Peak-to-Average Power Ratio of Orthogonal Frequency Division Multiplexing signal through bit of symbol interleaving", Elec. Letters, vol. 36, no. 13, pp. 1161-1162, June 2000.

[9] B. S. Krongold and D. L. Jones, "PAR reduction in OFDM via Active Constellation Extension", IEEE Trans. on Broadcasting, vol. 49, no. 3, pp. 258-268, Sept. 2003.

[10] 2006J. Tellado-Mourelo, "Peak-to-Average Power Ratio reduction for multicarrier Modulation”, PhD thesis, Stanford University, Sept. 1999.

[11] I. M. Mahafeno, Y. Louet and J.-F. Helard, "Peak-to-average power ratio reduction using second order cone programming based tone reservation for terrestrial digital video broadcasting systems for Terestrial DVB Systems", IET Communications, vol. 3, no. 7, pp. 12501261, July 2009.

[12] M. J. F.-G. Garcia, O. Edfors and J. M. Paez-Borrallo, "Peak Power Reduction for OFDM Systems with Orthogonal Pilot Sequences", IEEE Trans. on Wireless Com., vol. 5, no. 1, pp. 47-51, Jan. 2006.

[13] M. Sharif, M. Gharavi-Alkhansari and B. H. Khalaj, "On the peak-toaverage power of OFDM signals based on oversampling", IEEE trans. on Com., vol. 51, no. 1, pp. 72-78, Jan. 2003.

[14] R. Van Nee and A. de Wild, "Reducing the peak-to-average power ratio of OFDM", 48 IE IEE Vehicular Technology Conference, vol. 3, pp. 2072-2076, Ottawa Canada, May 1998. 